\title{
Radio emission of SN1993J: the complete picture
}

\section{Re-analysis of all the available VLBI data}

\author{
I. Martí-Vidal ${ }^{1,2}$, J. M. Marcaide ${ }^{1}$, A. Alberdi ${ }^{3}$, J. C. Guirado ${ }^{1}$, M. A. Pérez-Torres ${ }^{3}$, and E. Ros ${ }^{2,1}$ \\ 1 Dpt. Astronomia i Astrofísica, Universitat de València, C/ Dr. Moliner 50, 46100 Burjassot, Spain \\ e-mail: imartiv@mpifr-bonn.mpg.de \\ 2 Max-Planck-Institut für Radioastronomie, Auf dem Hügel 69, 53121 Bonn, Germany \\ 3 Instituto de Astrofísica de Andalucía (CSIC), C/ Camino bajo de Huétor 50, 18008 Granada, Spain
}

Received 9 December 2009 / Accepted 29 October 2010

\begin{abstract}
We have performed a complete re-calibration and re-analysis of all the available Very-Long-Baseline-Interferometry (VLBI) observations of supernova SN1993J, following an homogeneous and well-defined methodology. VLBI observations of SN1993J at 69 epochs, spanning 13 years, were performed by two teams, which used different strategies and analysis tools. The results obtained by each group are similar, but their conclusions on the supernova expansion and the shape and evolution of the emitting region differ significantly. From our analysis of the combined set of observations, we have obtained an expansion curve with unprecedented time resolution and coverage. We find that the data from both teams are compatible when analyzed with the same methodology. One expansion index $\left(m_{3}=0.87 \pm 0.02\right)$ is enough to model the expansion observed at $1.7 \mathrm{GHz}$, while two expansion indices $\left(m_{1}=0.933 \pm 0.010\right.$ and $m_{2}=0.796 \pm 0.005$ ), separated by a break time, $t_{\mathrm{br}}=390 \pm 30$ days, are needed to model the data, at frequencies higher than $1.7 \mathrm{GHz}$, up to day 4000 after explosion. We thus confirm the wavelength dependence of the size of the emitting region reported by one of the groups. We also find that all sizes measured at epochs later than day 4000 after explosion are systematically smaller than our model predictions (i.e., an additional expansion index might be needed to properly model these data). We also estimate the fractional shell width $(0.31 \pm 0.02$, average of all epochs and frequencies $)$ and the level of opacity to the radio emission by the ejecta. We find evidence of a spectral-index radial gradient in the supernova shell, which is indicative of a frequency-dependent ejecta opacity. Finally, we study the distribution and evolution of the azimuthal anisotropies (hot spots) found around the radio shell during the expansion. These anisotropies have intensities of $\sim 20 \%$ of the mean flux density of the shell, and appear to systematically evolve during the expansion.
\end{abstract}

Key words. radio continuum: stars - supernovae: general - supernovae: individual: SN1993J - galaxies: individual: M 81

\section{Introduction}

Supernova SN 1993J, in the galaxy M 81, has been one of the brightest supernovae ever in the radio band. The peak of emission at $5 \mathrm{GHz}$ was $\sim 100 \mathrm{mJy}$ (e.g. Weiler et al. 2007), much larger than typical peak flux densities of radio supernovae. The large flux density of this supernova, together with its high declination, allowed for long observing campaigns with VLBI. Two research groups (one led by Bartel and the other one led by Marcaide) have monitored this supernova with the VLBI technique, from 1993 (Marcaide et al. 1994; Bartel et al. 1994) to 2005 .

Different results on the structure and expansion of the radioemitting region of SN 1993J have been reported by both groups, based on the subset of VLBI data taken by each group. Marcaide et al. (1997) reported the first evidence of deceleration in the shell expansion (i.e., $R \propto t^{m}$, see Chevalier 1982a), with an estimated expansion index of $m=0.86 \pm 0.02$. Bartel et al. (2002) confirmed a deceleration, but claimed up to four changes in the value of $m$ corresponding to four different expansion periods and interpreted the changes in the expansion index as changes in the mass-loss wind of the progenitor star through the pre-supernova stage. However, from their set of VLBI observations, which range from day 182 to day 3867 after explosion, Marcaide et al. (2009) found a wavelength-dependent expansion curve that can be modeled using only one expansion index $(m=0.86)$ for their low-frequency data data $(1.7 \mathrm{GHz})$ and two expansion indices ( 0.86 and 0.79 , separated by a break time on day $\sim 1500$ after explosion) for the data at all higher frequencies. These authors interpret the frequency-dependent expansion curve as being caused by (the possible combination of) two effects: 1) a changing (and frequency-dependent) opacity to the radio emission by the supernova ejecta ${ }^{1}$ and 2) a radial drop in the amplified magnetic fields inside the radiating region combined with the finite sensitivity of the VLBI observations.

In this paper (Paper I), we report on a homogeneous analysis of the complete set of available VLBI observations of SN 1993J (69 epochs), using different approaches to minimizing the effects of any possible bias in the data analysis. We studied the details of the expansion curve at several frequencies and the evolution of the structure of the radio shell throughout the history of the SN 1993J radio emission, with unprecedented time resolution and coverage. We confirm earlier findings reported in Marcaide et al. (2009) and report a model of the expansion curve compatible with the shell sizes obtained using different approaches. We also present a study of the distribution and

\footnotetext{
1 The ejecta are located behind the inner boundary of the radio shell and may block (partly or fully) the emission coming the backside of the shell; see Eq. (B.1) of Marcaide et al. (2009) for a mathematical definition of the ejecta opacity in our shell model.
} 
evolution of inhomogeneities inside the shell. In another publication (Martí-Vidal et al. 2011, hereafter Paper II), we present a new simulation code able to simultaneously model the expansion curve and the radio light curves of SN 1993J reported by Weiler et al. (2007). We then present the extensions to the Chevalier model (Chevalier 1982a,b) to satisfactorily fit all the radio data.

In Sect. 2 we describe the complete set of VLBI observations of SN 1993J, most of which we have re-analyzed ab initio. In Sect. 3.1 we report on the location and proper motion of the radio shell. In Sect. 3.2 we report on the complete expansion curve, obtained with different approaches, and in Sect. 3.4 we discuss the departure in the evolution of the supernova structure from self-similarity.

\section{Observations}

In Table 1 we show the complete set of available VLBI observations of SN 1993J, made from year 1993 through the end of year 2005. There is a total of 69 observing epochs, many of them made at several frequencies. All these observations used global VLBI arrays. In nearly all of them (except for some epochs in 1993 and 1994), the whole VLBA (10 identical antennas of $25 \mathrm{~m}$ diameter spread over the USA) and the Phased-VLA (equivalent to a $\sim 130 \mathrm{~m}$ antenna in New Mexico, USA) were used. Other antennas observed less often (each antenna participated in around 50\% of the epochs): Green Bank $(110 \times 100 \mathrm{~m}$, West Virginia) and Goldstone (70 $\mathrm{m}$, California) in the USA, and part of the European VLBI Network (Effelsberg, $100 \mathrm{~m}$, Germany; Medicina, $32 \mathrm{~m}$, Italy; Noto, $32 \mathrm{~m}$, Italy; Jodrell Bank, $76 \mathrm{~m}$, UK; Onsala, 25 m, Sweden; Westerbork, 93 m, The Netherlands; and Robledo, $70 \mathrm{~m}$, Spain). The arrays typically consisted of about 15 antennas at each observing epoch, with the exception of the first 4 epochs of Marcaide's group (between 1993 and 1994, see Table 1) with less than 6 antennas.

At each epoch, the observations typically lasted between 12 and $16 \mathrm{~h}$ (again, with the exception of some shorter runs of Marcaide's group in 1993 and 1994). The observations were taken in a phase-reference manner, with the exception of the first ten epochs of Marcaide's group (the supernova was well detected in all baselines at these epochs). Scans of the radio core of source M 81 (hereafter, M 81*) were inserted between the scans of the supernova, with duty cycles a few minutes long (from $\sim 2$ to $\sim 10 \mathrm{~min}$, depending on epoch and observing frequency). Fringe finders and flux calibrators, both primary and secondary, were usually observed a few times during each epoch (depending on epoch, the sources 3C 286, 3C 48, B0917+624, B0954+658, and OQ 208 were observed).

Additional technical details on these observations can be found in Marcaide et al. (1997, 2009), Bartel et al. (2002), Martí-Vidal (2008), and references therein.

\subsection{Data calibration and imaging}

We completely re-calibrated (in amplitude, delay, and delay rate) all the available epochs of Bartel's group that were observed after 1995, and all our own phase-referenced epochs, following a uniform strategy. For the epochs earlier than 1995 that were observed by Bartel's group, we adapted the results published in Bartel et al. (2002) to our analysis strategy, as we explain in Sect. 3.2. For the visibility calibration, we used the NRAO Astronomical Image Processing System (AIPS) ${ }^{2}$. We first aligned the visibility phases through all the frequency bands (for all

\footnotetext{
$\overline{2}$ http://www.aips.nrao.edu
}

sources and times) by fringe-fitting the single-band delays of one scan of a fringe finder, or flux calibrator, and then applying the estimated antenna delays and phases to all visibilities. Then, a second fringe-fitting, now using the multiband delays, provided the new phase, delay, and rate corrections to all the observations. We performed the visibility amplitude calibration using system temperatures and gain curves from each antenna. We then transferred the calibrated visibilities of M 81* into the program DIFMAP ${ }^{3}$ (Shepherd et al. 1995) and made several iterations of phase and gain self-calibration until we obtained a highquality image of M 81*. The M 81* CLEAN model obtained in DIFMAP was used again in AIPS for another fringe-fitting iteration of the $\mathrm{M} 81^{*}$ data. Therefore, the new estimated antenna phases, delays, and rates were free of the (small) structure contributions of M $81 *$. Such antenna corrections were then interpolated in time and applied to the SN 1993J visibilities using the AMBG option of the AIPS task CLCAL ${ }^{4}$. The amplitudes of the antenna gains were refined using the CLEAN model of M 81* with the AIPS task CALIB. These corrections were also interpolated and applied to the scans of SN 1993J. For the case of Bartel's group observations, we did not apply any calibration to the cross-polarization data, and all our images and fits were performed using the Stokes $I$ data (i.e., total intensity of the source, RCP + LCP flux densities). At this stage, we edited bad visibilities based on standard selection criteria.

The process of imaging the supernova was performed following the special procedure described in Marcaide et al. (2009), but re-centering the supernova shell (at each frequency and epoch) according to the shifts reported in the next section. The most important details related to the imaging procedure described in Marcaide et al. (2009) are 1) use of a dynamic Gaussian taper in Fourier space prior to the deconvolution (to avoid possible resolution artifacts) and 2) phase self-calibration restricted to the shortest baselines, taking advantage of the source azimuthal symmetry. To check for any possible effect coming from this calibration procedure, we repeated all the analyses described in the following sections using the phasereferenced visibilities directly, without any further calibration.

We notice that the visibilities of epochs observed at $2.3 \mathrm{GHz}$ are noisier than those of the other epochs. The $u v$-coverages of most of these epochs are also poor. Therefore, the quality of the results obtained at $2.3 \mathrm{GHz}$ is worse (see the dynamic ranges of all epochs in Table 1). In any case, the results at $2.3 \mathrm{GHz}$ are consistent with those obtained at the other frequencies.

\section{Data analysis}

\subsection{Location and proper motion of the expansion center}

The first step in the analysis process was to precisely determine the location of the supernova geometric center (i.e., the center of the shell-like structure) and its possible evolution in time. This step is essential for a correct determination of the shell size (and width), since a systematic offset of the fitted models from the real shell center would translate into biases in the analysis strategies reported in this paper. To locate the supernova center, we fitted a simplified shell model to the visibilities. Such a model consisted of a homogeneous, optically-thin, spherical shell of outer radius $R_{\text {out }}$ and inner radius $R_{\text {in }}$ with a variable degree of central

\footnotetext{
3 ftp://ftp.astro.caltech.edu/pub/difmap

4 Using this option, AIPS tries to find out the possible $2 \pi$ phase cycles introduced in the residuals between the scans of the calibrator source, correcting the phase interpolations of the target source scans.
} 
I. Martí-Vidal et al.: Radio emission of SN1993J: the complete picture. I.

Table 1. VLBI observations of SN 1993J.

\begin{tabular}{|c|c|c|c|c|c|c|c|}
\hline $\begin{array}{l}\text { Date } \\
\text { (dd/mm/yy) }\end{array}$ & $\begin{array}{l}\text { Age }^{1} \\
\text { (days) }\end{array}$ & $\overline{\mathrm{PI}^{2}}$ & $\begin{array}{l}\text { Freq. } \\
(\mathrm{GHz})\end{array}$ & $\begin{array}{l}R_{\mathrm{SC}}{ }^{3} \\
(\mathrm{mas})\end{array}$ & $\begin{array}{l}R_{\mathrm{PR}}{ }^{4} \\
(\mathrm{mas})\end{array}$ & $\begin{array}{l}R_{\mathrm{MF}}{ }^{5} \\
(\mathrm{mas})\end{array}$ & 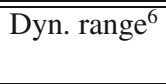 \\
\hline $27 / 04 / 93^{7}$ & 30 & $\mathrm{~B}$ & 22 & $0.094 \pm 0.004$ & $0.094 \pm 0.004$ & $0.089 \pm 0.008$ & $\ldots$ \\
\hline \multirow[t]{3}{*}{$17 / 05 / 93^{7}$} & 50 & B & 22 & $0.154 \pm 0.005$ & $0.154 \pm 0.005$ & $0.146 \pm 0.010$ & $\ldots$ \\
\hline & & & 15 & $0.162 \pm 0.018$ & $0.162 \pm 0.018$ & $0.15 \pm 0.03$ & $\ldots$ \\
\hline & & & 8.4 & $0.134 \pm 0.005$ & $0.134 \pm 0.005$ & $0.127 \pm 0.009$ & $\ldots$ \\
\hline \multirow[t]{2}{*}{$27 / 06 / 93^{7}$} & 91 & B & 15 & $0.254 \pm 0.008$ & $0.254 \pm 0.008$ & $0.241 \pm 0.015$ & $\ldots$ \\
\hline & & & 8.4 & $0.264 \pm 0.006$ & $0.264 \pm 0.006$ & $0.250 \pm 0.010$ & $\ldots$ \\
\hline \multirow[t]{2}{*}{$04 / 08 / 93^{7}$} & 129 & B & 15 & $0.38 \pm 0.03$ & $0.38 \pm 0.03$ & $0.36 \pm 0.06$ & $\ldots$ \\
\hline & & & 8.4 & $0.347 \pm 0.008$ & $0.347 \pm 0.009$ & $0.329 \pm 0.015$ & $\ldots$ \\
\hline \multirow[t]{2}{*}{$19 / 09 / 93^{7}$} & 175 & B & 15 & $0.461 \pm 0.008$ & $0.461 \pm 0.008$ & $0.437 \pm 0.015$ & $\ldots$ \\
\hline & & & 8.4 & $0.448 \pm 0.008$ & $0.448 \pm 0.009$ & $0.425 \pm 0.015$ & $\ldots$ \\
\hline $26 / 09 / 93^{8}$ & 182 & M & 8.4 & $0.517 \pm 0.017$ & $0.517 \pm 0.018$ & $0.49 \pm 0.03$ & 307.4 \\
\hline \multirow[t]{3}{*}{$06 / 11 / 93^{7}$} & 223 & $\mathrm{~B}$ & 15 & $0.565 \pm 0.015$ & $0.565 \pm 0.015$ & $0.54 \pm 0.03$ & $\ldots$ \\
\hline & & & 8.4 & $0.561 \pm 0.008$ & $0.561 \pm 0.009$ & $0.531 \pm 0.015$ & $\ldots$ \\
\hline & & & 5.0 & $0.565 \pm 0.017$ & $0.565 \pm 0.017$ & $0.54 \pm 0.03$ & $\ldots$ \\
\hline $22 / 11 / 93^{8}$ & 239 & M & 8.4 & $0.67 \pm 0.06$ & $0.67 \pm 0.06$ & $0.63 \pm 0.11$ & 450.9 \\
\hline \multirow[t]{2}{*}{$17 / 12 / 93^{7}$} & 264 & $\mathrm{~B}$ & 15 & $0.77 \pm 0.03$ & $0.77 \pm 0.03$ & $0.73 \pm 0.05$ & $\ldots$ \\
\hline & & & 8.4 & $0.710 \pm 0.011$ & $0.710 \pm 0.011$ & $0.673 \pm 0.019$ & $\ldots$ \\
\hline \multirow[t]{3}{*}{$28 / 01 / 94^{7}$} & 306 & B & 15 & $0.73 \pm 0.04$ & $0.73 \pm 0.04$ & $0.69 \pm 0.07$ & $\ldots$ \\
\hline & & & 8.4 & $0.793 \pm 0.014$ & $0.793 \pm 0.014$ & $0.75 \pm 0.02$ & $\ldots$ \\
\hline & & & 5.0 & $0.78 \pm 0.02$ & $0.78 \pm 0.02$ & $0.74 \pm 0.04$ & $\ldots$ \\
\hline $20 / 02 / 94^{8}$ & 329 & M & 8.4 & $0.87 \pm 0.07$ & $0.87 \pm 0.07$ & $0.82 \pm 0.12$ & 199.3 \\
\hline \multirow[t]{2}{*}{$15 / 03 / 94^{7}$} & 352 & $\mathrm{~B}$ & 8.4 & $0.862 \pm 0.014$ & $0.862 \pm 0.014$ & $0.82 \pm 0.02$ & $\ldots$ \\
\hline & & & 5.0 & $0.863 \pm 0.014$ & $0.863 \pm 0.014$ & $0.82 \pm 0.02$ & $\ldots$ \\
\hline \multirow[t]{3}{*}{$22 / 04 / 94^{7}$} & 390 & B & 8.4 & $0.991 \pm 0.017$ & $0.991 \pm 0.017$ & $0.94 \pm 0.03$ & $\ldots$ \\
\hline & & & 5.0 & $0.955 \pm 0.017$ & $0.955 \pm 0.017$ & $0.90 \pm 0.03$ & $\ldots$ \\
\hline & & & 2.3 & $1.00 \pm 0.05$ & $1.00 \pm 0.05$ & $0.95 \pm 0.09$ & $\ldots$ \\
\hline $29 / 05 / 94^{8}$ & 427 & M & 8.4 & $1.08 \pm 0.10$ & $1.08 \pm 0.11$ & $1.02 \pm 0.18$ & 123.8 \\
\hline \multirow{2}{*}{$22 / 06 / 94^{7}$} & 451 & $\mathrm{~B}$ & 8.4 & $1.062 \pm 0.017$ & $1.062 \pm 0.017$ & $1.01 \pm 0.03$ & $\ldots$ \\
\hline & & & 5.0 & $1.098 \pm 0.017$ & $1.098 \pm 0.017$ & $1.04 \pm 0.03$ & $\ldots$ \\
\hline \multirow[t]{3}{*}{$30 / 08 / 94^{7}$} & 520 & B & 8.4 & $1.248 \pm 0.019$ & $1.25 \pm 0.02$ & $1.18 \pm 0.03$ & $\ldots$ \\
\hline & & & 5.0 & $1.232 \pm 0.019$ & $1.23 \pm 0.02$ & $1.17 \pm 0.03$ & $\ldots$ \\
\hline & & & 2.3 & $1.29 \pm 0.04$ & $1.29 \pm 0.04$ & $1.22 \pm 0.08$ & $\ldots$ \\
\hline $20 / 09 / 94^{8}$ & 541 & M & 5.0 & $1.21 \pm 0.14$ & $1.21 \pm 0.14$ & $1.15 \pm 0.24$ & 152.4 \\
\hline \multirow[t]{2}{*}{$31 / 10 / 94^{7}$} & 582 & $\mathrm{~B}$ & 8.4 & $1.35 \pm 0.02$ & $1.35 \pm 0.02$ & $1.28 \pm 0.04$ & $\ldots$ \\
\hline & & & 5.0 & $1.35 \pm 0.02$ & $1.35 \pm 0.02$ & $1.27 \pm 0.04$ & $\ldots$ \\
\hline \multirow[t]{3}{*}{$23 / 12 / 94^{7}$} & 635 & B & 8.4 & $1.39 \pm 0.02$ & $1.39 \pm 0.02$ & $1.31 \pm 0.04$ & $\ldots$ \\
\hline & & & 5.0 & $1.43 \pm 0.02$ & $1.43 \pm 0.02$ & $1.36 \pm 0.04$ & $\ldots$ \\
\hline & & & 2.3 & $1.48 \pm 0.05$ & $1.48 \pm 0.05$ & $1.40 \pm 0.09$ & $\ldots$ \\
\hline $12 / 02 / 95$ & 686 & B & 8.4 & $1.52 \pm 0.08$ & $1.53 \pm 0.08$ & $1.41 \pm 0.04$ & 42.7 \\
\hline $23 / 02 / 95$ & 697 & M & 5.0 & $1.48 \pm 0.04$ & $1.48 \pm 0.04$ & $1.38 \pm 0.11$ & 140.7 \\
\hline \multirow[t]{2}{*}{$11 / 05 / 95$} & 774 & B & 8.4 & $1.60 \pm 0.20$ & $1.64 \pm 0.21$ & $1.68 \pm 0.12$ & 36.9 \\
\hline & & & 5.0 & $1.68 \pm 0.10$ & $1.68 \pm 0.11$ & $1.56 \pm 0.04$ & 14.2 \\
\hline $11 / 05 / 95$ & 774 & M & 5.0 & $1.66 \pm 0.07$ & $1.71 \pm 0.08$ & $1.51 \pm 0.80$ & 194.6 \\
\hline \multirow[t]{2}{*}{$18 / 08 / 95$} & 873 & B & 8.4 & $1.73 \pm 0.16$ & $1.73 \pm 0.16$ & $1.77 \pm 0.11$ & 23.5 \\
\hline & & & 5.0 & $1.85 \pm 0.07$ & $1.81 \pm 0.07$ & $1.51 \pm 0.30$ & 44.5 \\
\hline 01/10/95 & 917 & M & 5.0 & $1.92 \pm 0.07$ & $1.92 \pm 0.08$ & $1.92 \pm 0.05$ & 168.7 \\
\hline $19 / 12 / 95$ & 996 & B & 8.4 & $2.11 \pm 0.06$ & $2.11 \pm 0.06$ & $2.12 \pm 0.11$ & 35.2 \\
\hline & & & 5.0 & $2.15 \pm 0.11$ & $2.15 \pm 0.12$ & $2.13 \pm 0.07$ & 71.0 \\
\hline & & & 2.3 & $2.23 \pm 0.05$ & $2.23 \pm 0.05$ & $2.11 \pm 0.08$ & 116.9 \\
\hline 28/03/96 & 1096 & M & 5.0 & $2.21 \pm 0.05$ & $2.21 \pm 0.06$ & $2.14 \pm 0.11$ & 63.0 \\
\hline 08/04/96 & 1107 & $\mathrm{~B}$ & 8.4 & $2.41 \pm 0.18$ & $2.34 \pm 0.19$ & $2.14 \pm 0.15$ & 52.3 \\
\hline & & & 5.0 & $2.10 \pm 0.20$ & $2.02 \pm 0.21$ & $1.98 \pm 0.12$ & 56.0 \\
\hline $17 / 06 / 96$ & 1177 & M & 5.0 & $2.31 \pm 0.07$ & $2.31 \pm 0.07$ & $2.23 \pm 0.10$ & 30.6 \\
\hline $01 / 09 / 96$ & 1253 & $\mathrm{~B}$ & 8.4 & $2.50 \pm 0.10$ & $2.53 \pm 0.10$ & $2.29 \pm 0.06$ & 30.7 \\
\hline & & & 5.0 & $2.51 \pm 0.08$ & $2.50 \pm 0.08$ & $2.33 \pm 0.04$ & 74.5 \\
\hline $22 / 10 / 96$ & 1304 & M & 5.0 & $2.61 \pm 0.04$ & $2.61 \pm 0.04$ & $2.47 \pm 0.06$ & 120.9 \\
\hline $13 / 12 / 96$ & 1356 & B & 8.4 & $2.65 \pm 0.06$ & $2.66 \pm 0.06$ & $2.52 \pm 0.07$ & 20.6 \\
\hline & & & 5.0 & $2.62 \pm 0.11$ & $2.63 \pm 0.12$ & $2.59 \pm 0.11$ & 62.8 \\
\hline & & & 2.3 & $2.52 \pm 0.18$ & $2.52 \pm 0.19$ & $2.44 \pm 0.10$ & 165.1 \\
\hline
\end{tabular}


Table 1. continued.

\begin{tabular}{|c|c|c|c|c|c|c|c|}
\hline $\begin{array}{l}\text { Date } \\
(\mathrm{dd} / \mathrm{mm} / \mathrm{yy})\end{array}$ & $\begin{array}{l}\text { Age }^{1} \\
\text { (days) }\end{array}$ & $\overline{\mathrm{PI}^{2}}$ & $\begin{array}{l}\text { Freq. } \\
(\mathrm{GHz})\end{array}$ & $\begin{array}{l}R_{\mathrm{SC}}{ }^{3} \\
(\mathrm{mas})\end{array}$ & $\begin{array}{l}R_{\mathrm{PR}}{ }^{4} \\
(\mathrm{mas})\end{array}$ & $\begin{array}{l}R_{\mathrm{MF}}^{5} \\
(\mathrm{mas})\end{array}$ & Dyn. range $^{6}$ \\
\hline $25 / 02 / 97$ & 1430 & $\mathrm{M}$ & 5.0 & $2.81 \pm 0.09$ & $2.81 \pm 0.10$ & $2.51 \pm 0.10$ & 79.4 \\
\hline \multirow[t]{2}{*}{ 07/06/97 } & 1532 & B & 8.4 & $2.90 \pm 0.13$ & $2.90 \pm 0.14$ & $2.73 \pm 0.11$ & 19.1 \\
\hline & & & 5.0 & $2.86 \pm 0.06$ & $2.84 \pm 0.07$ & $2.70 \pm 0.43$ & 21.6 \\
\hline $21 / 09 / 97$ & 1638 & M & 5.0 & $3.09 \pm 0.05$ & $3.09 \pm 0.06$ & $2.94 \pm 0.02$ & 101.9 \\
\hline \multirow[t]{3}{*}{$15 / 11 / 97$} & 1693 & B & 8.4 & $3.14 \pm 0.07$ & $3.15 \pm 0.12$ & $2.91 \pm 0.15$ & 31.2 \\
\hline & & & 5.0 & $3.13 \pm 0.15$ & $3.13 \pm 0.16$ & $3.05 \pm 0.13$ & 84.0 \\
\hline & & & 2.3 & $3.17 \pm 0.50$ & $3.17 \pm 0.57$ & $3.03 \pm 0.16$ & 113.6 \\
\hline $18 / 02 / 98$ & 1788 & M & 5.0 & $3.37 \pm 0.05$ & $3.39 \pm 0.05$ & $3.21 \pm 0.05$ & 86.6 \\
\hline $30 / 05 / 98$ & 1889 & M & 5.0 & $3.48 \pm 0.06$ & $3.48 \pm 0.07$ & $3.33 \pm 0.09$ & 112.6 \\
\hline \multirow[t]{2}{*}{ 03/06/98 } & 1893 & B & 8.4 & $3.46 \pm 0.07$ & $3.37 \pm 0.08$ & $3.33 \pm 0.14$ & 35.4 \\
\hline & & & 5.0 & $3.43 \pm 0.09$ & $3.45 \pm 0.09$ & $3.18 \pm 0.13$ & 26.9 \\
\hline 20/11/98 & 2064 & B & 5.0 & $3.73 \pm 0.16$ & $3.75 \pm 0.17$ & $3.63 \pm 0.06$ & 26.0 \\
\hline $23 / 11 / 98$ & 2066 & M & 5.0 & $3.74 \pm 0.07$ & $3.74 \pm 0.08$ & $3.50 \pm 0.05$ & 94.7 \\
\hline $30 / 11 / 98$ & 2073 & M & 1.7 & $3.78 \pm 0.07$ & $3.91 \pm 0.08$ & $3.58 \pm 0.15$ & 150.5 \\
\hline \multirow[t]{2}{*}{$07 / 12 / 98$} & 2080 & B & 8.4 & $3.74 \pm 0.09$ & $3.75 \pm 0.09$ & $3.47 \pm 0.14$ & 30.0 \\
\hline & & & 2.3 & $3.77 \pm 0.07$ & $3.96 \pm 0.08$ & $3.57 \pm 0.16$ & 62.0 \\
\hline 06/06/99 & 2261 & B & 1.7 & $4.11 \pm 0.07$ & $4.17 \pm 0.08$ & $3.82 \pm 0.05$ & 60.1 \\
\hline $10 / 06 / 99$ & 2265 & M & 5.0 & $4.07 \pm 0.08$ & $4.10 \pm 0.08$ & $3.81 \pm 0.04$ & 61.9 \\
\hline $16 / 06 / 99$ & 2271 & B & 5.0 & $4.05 \pm 0.35$ & $4.02 \pm 0.36$ & $3.71 \pm 0.16$ & 23.3 \\
\hline 22/09/99 & 2369 & M & 5.0 & $4.12 \pm 0.07$ & $4.12 \pm 0.08$ & $3.93 \pm 0.05$ & 41.1 \\
\hline 28/09/99 & 2376 & B & 1.7 & $4.28 \pm 0.07$ & $4.43 \pm 0.08$ & $4.13 \pm 0.09$ & 3.8 \\
\hline $24 / 11 / 99$ & 2432 & B & 5.0 & $4.32 \pm 0.22$ & $4.34 \pm 0.23$ & $4.12 \pm 0.12$ & 21.8 \\
\hline $25 / 02 / 00$ & 2525 & B & 8.4 & $4.43 \pm 0.09$ & $4.48 \pm 0.10$ & $4.12 \pm 0.32$ & 26.9 \\
\hline 06/06/00 & 2627 & M & 5.0 & $4.52 \pm 0.10$ & $4.38 \pm 0.11$ & $4.36 \pm 0.06$ & 40.2 \\
\hline \multirow[t]{2}{*}{$13 / 11 / 00$} & 2787 & B & 8.4 & $4.77 \pm 0.13$ & $4.65 \pm 0.13$ & $4.90 \pm 0.29$ & 14.5 \\
\hline & & & 2.3 & $4.85 \pm 0.40$ & $4.70 \pm 0.39$ & $4.81 \pm 0.25$ & 19.5 \\
\hline $20 / 11 / 00$ & 2794 & M & 1.7 & $5.01 \pm 0.13$ & $5.07 \pm 0.13$ & $4.75 \pm 0.05$ & 219.7 \\
\hline $24 / 11 / 00$ & 2798 & M & 5.0 & $4.76 \pm 0.18$ & $4.79 \pm 0.18$ & $4.76 \pm 0.06$ & 56.71 \\
\hline $14 / 02 / 01$ & 2880 & M & 5.0 & $4.94 \pm 0.12$ & $4.95 \pm 0.12$ & $4.65 \pm 0.07$ & 116.2 \\
\hline $10 / 06 / 01$ & 2996 & B & 5.0 & $5.00 \pm 0.14$ & $4.99 \pm 0.14$ & $4.90 \pm 0.09$ & 32.1 \\
\hline $18 / 11 / 01$ & 3157 & M & 5.0 & $5.21 \pm 0.18$ & $5.16 \pm 0.19$ & $4.28 \pm 0.60$ & 38.7 \\
\hline $26 / 11 / 01$ & 3164 & B & 1.7 & $5.59 \pm 0.17$ & $5.52 \pm 0.18$ & $5.17 \pm 0.12$ & 98.1 \\
\hline $24 / 05 / 02$ & 3344 & B & 5.0 & $5.49 \pm 0.18$ & $5.48 \pm 0.19$ & $5.03 \pm 0.06$ & 13.2 \\
\hline 07/11/02 & 3511 & M & 5.0 & $5.77 \pm 0.19$ & $5.76 \pm 0.19$ & $5.57 \pm 0.40$ & 18.2 \\
\hline $17 / 11 / 02$ & 3521 & M & 1.7 & $6.15 \pm 0.18$ & $6.06 \pm 0.19$ & $5.85 \pm 0.10$ & 78.5 \\
\hline $01 / 06 / 03$ & 3717 & B & 5.0 & $6.04 \pm 0.23$ & $5.79 \pm 0.23$ & $5.26 \pm 0.29$ & 19.6 \\
\hline 29/10/03 & 3867 & M & 5.0 & $6.34 \pm 0.18$ & $6.34 \pm 0.20$ & $5.88 \pm 0.22$ & 21.9 \\
\hline $25 / 05 / 04$ & 4076 & B & 5.0 & $6.45 \pm 0.31$ & $6.13 \pm 0.32$ & $5.74 \pm 0.30$ & 15.3 \\
\hline 06/06/04 & 4088 & B & 1.7 & $6.72 \pm 0.21$ & $6.58 \pm 0.24$ & $6.49 \pm 0.14$ & 35.4 \\
\hline $31 / 10 / 04$ & 4235 & M & 5.0 & $6.33 \pm 0.24$ & $6.56 \pm 0.24$ & $\ldots 9$ & 19.8 \\
\hline $11 / 06 / 05$ & 4458 & B & 5.0 & $6.55 \pm 0.26$ & $6.89 \pm 0.27$ & $5.90 \pm 0.61$ & 18.7 \\
\hline $22 / 10 / 05$ & 4591 & M & 5.0 & $6.64 \pm 0.29$ & $6.64 \pm 0.29$ & $6.10 \pm 0.57$ & 27.3 \\
\hline $06 / 11 / 05$ & 4606 & M & 1.7 & $7.00 \pm 0.30$ & $6.87 \pm 0.27$ & $6.58 \pm 0.11$ & 45.9 \\
\hline
\end{tabular}

Notes. ${ }^{(1)}$ Assumed explosion date: 28 March 1993. ${ }^{(2)}$ Principal Investigator: $\quad$ M = J. M. Marcaide; $\quad$ B = N. Bartel / M. F. Bietenholz / M. Rupen. (3) Shell radii determined with the CPM applied to images obtained from self-calibrated visibilities (see text). ${ }^{(4)}$ Shell radii determined with the CPM applied to images obtained from phase-referenced visibilities (see text). ${ }^{(5)}$ Shell radii determined from model fitting to the visibilities. (6) Dynamic range of the supernova images (i.e., peak flux density in units of the root-mean-square of the image background), computed using natural visibility weighting and applying a Gaussian taper in Fourier space (see Sect. 2.1). ${ }^{(7)}$ Epochs where the shell radii were taken from Bartel et al. (2002), but applying the corresponding biases (see Sect. 3.2.1) to make them comparable to the radii obtained with the CPM and with the radii estimated from our visibility model fitting. ${ }^{(8)}$ Epochs where the "CPM-like" radii were adapted from model-fitting results, to avoid image over-resolutions if the CPM were applied directly. ${ }^{(9)}$ Unsatisfactory fit (unclear minimum of the $\chi^{2}$ ).

absorption by the ejecta (i.e., with partial blockage of the emission from the rear side of the shell for radii $\left.<R_{\text {in }}\right)$.

Previous analyses of SN 1993J data (Marcaide et al. 2005; Bietenholz et al. 2003), each based on a different approach, have concluded that there must be some opacity to the radio emission by the ejecta material. Marcaide et al. (2005) conclude, from their Green-function deconvolution approach, that the ejecta opacity should be $100 \%$ at all wavelengths, at least for the epochs they analyzed. Marcaide et al. (2009) confirmed these results by studying the shapes of the azimuthal average of the supernova at different epochs and comparing them with several theoretical models. In contrast, Bietenholz et al. (2003) conclude that the ejecta absorption must be as small as $25 \%$. These authors used a simplified disk-like absorption pattern for the radio 

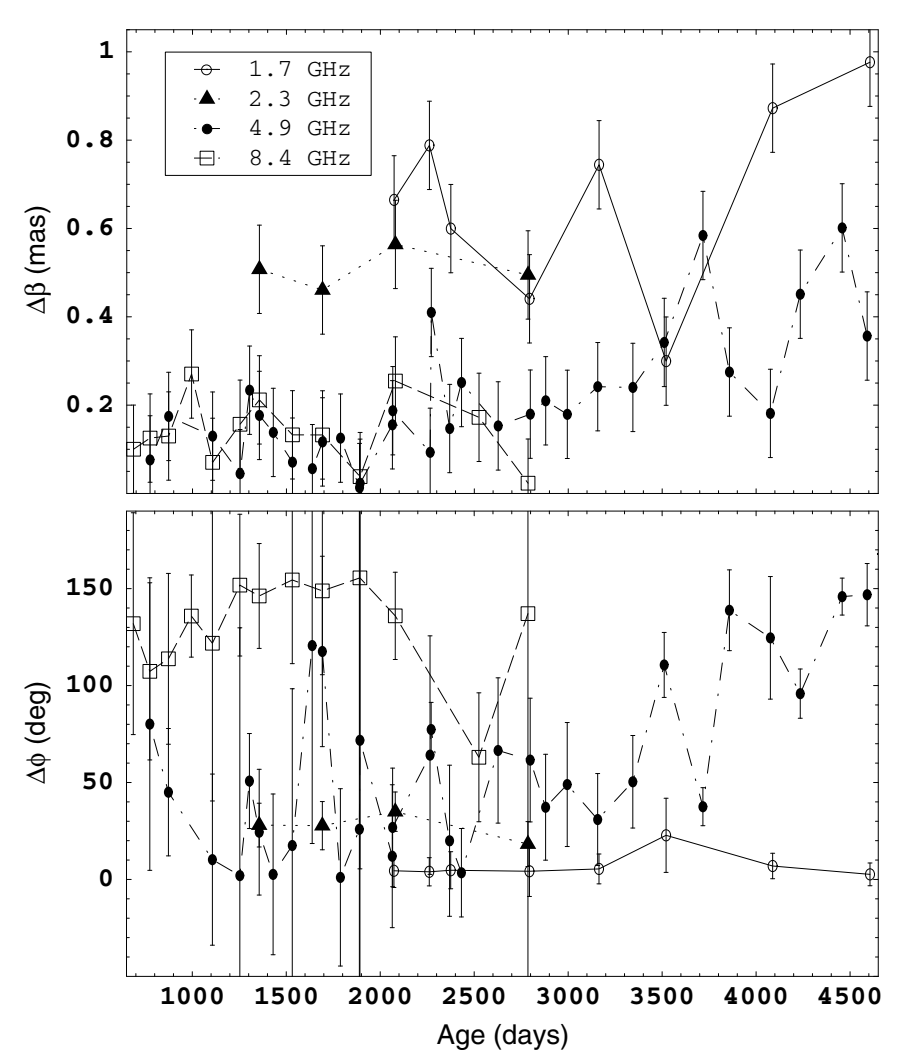

Fig. 1. Shifts in separation, $\beta$, and position angle, $\phi$, of the SN 1993J shell center (using M $81 *$ as phase calibrator) with respect to the SN 1993J position on day 1889 after explosion.

emission, a model we consider unrealistic. To accurately determine the location of the shell center, and to check for any possible biases coming from the use of different absorption models to fit the visibilities, we used a shell model with a variable degree of absorption from the ejecta and studied the effect of using different ejecta absorptions on the estimates of the shell center.

The Fourier transform of a shell model with absorption from the inner side does not have an analytical expression. Thus, we generated an interpolation function of such a Fourier transform, using the fractional shell width, $\xi=\left(R_{\text {out }}-R_{\text {in }}\right) / R_{\text {out }}$, the shell radius, $R_{\text {out }}$, the percentage of absorption, and the position of the shell center as interpolating variables for computating the $\chi^{2}$ of our fits. This model is essentially the same as the one described in Appendix 2 of Marcaide et al. (2009), but with the addition of a plane-wave phase factor to account for the shift of the shell in the sky plane. Fitting the visibilities to a shell model with maximum ejecta absorption and a relative shell width of 0.3 , we estimated the shell size and the position of the supernova shell center for all epochs observed since year 1995. We notice that changing the shell width by a $20 \%$ or removing the absorption by the ejecta from the model did not change the estimates of the coordinates of the shell center above the $1 \sigma$ level. The shifts obtained, taking the supernova position at $5 \mathrm{GHz}$ on day 1889 after explosion as reference, are shown in Fig. 1.

From this figure, we reach several conclusions. The first is that the location of the phase-calibrator source, M 81*, taking the shell center of SN 1993J as a position reference, depends on the observing frequency. In fact, we find an average shift of $0.62 \pm 0.04$ mas in $\alpha \cos \delta$ and $0.10 \pm 0.02$ mas in $\delta$ between the $\mathrm{M} 81^{*}$ positions at $5 \mathrm{GHz}$ and $1.7 \mathrm{GHz}$, the average shift between $5 \mathrm{GHz}$ and $2.3 \mathrm{GHz}$ is $0.40 \pm 0.04$ mas in $\alpha \cos \delta$ and
$0.2 \pm 0.1$ mas in $\delta$, and the shifts between $5 \mathrm{GHz}$ and $8.4 \mathrm{GHz}$ are smaller $(-0.13 \pm 0.3$ mas in $\alpha \cos \delta$ and $-0.01 \pm 0.03$ mas in $\delta)$.

According to the standard jet model (Blandford \& Königl 1979), there should indeed be spectral shifts in the brightness peaks of the VLBI core-jet structures due to the frequencydependent transition of the ejected material from optically thick to optically thin, for the synchrotron radiation, as first found by Marcaide \& Shapiro $(1983,1984)$ and later confirmed in many cases (e.g. Kovalev et al. 2007). Similar results of the spectral shift of M 81* were reported by Bietenholz et al. (2004), who have estimated the position of the real core (i.e., the central black hole) of $\mathrm{M} 81^{*}$ by finding a sharp bound in the radio emission at all frequencies, taking these shifts and the different sizes of the radio structures into account. Our in-depth analysis of the M 81* structure evolution and core location at several frequencies and epochs will be reported elsewhere.

Another conclusion extracted from Fig. 1 is that the location of the supernova shell center does not evolve in time with respect to the phase calibrator, at least at our level of precision. There is a hint of a drift at $5 \mathrm{GHz}$ beyond day $\sim 3000$, but the scatter of data at these epochs is also larger. The proper motion at each frequency, fitted from the position shifts shown in Fig. 1, is compatible with zero at a 1-sigma level. The most siginificant value of proper motion is obtained if we fit the $5 \mathrm{GHz}$ shifts from day 3000 onwards. In this case we obtain a proper motion with module $54 \pm 31 \mu \mathrm{as} \mathrm{yr}^{-1}$, which is compatible with zero at a 2-sigma level. Bietenholz et al. (2001) also studied the proper motion of the SN 1993J radio shell and arrived at the conclusion that it is compatible with zero at the level of precision achievable with VLBI. To take the shift in the peak of M 81* into account at different frequencies, we corrected the visibilities of SN 1993J at each epoch by applying the average shifts found in the SN 1993J images between frequencies, prior to any further data analysis. The center of the shell is thus a fixed parameter in all the analyses reported in the following sections ${ }^{5}$.

We notice that the scattering in the estimated positions of SN 1993J, shown in Fig. 1, may translate into an additional uncertainty in the estimate of the size of the supernova shell, since we consider the position of M 81* stationary with respect to the supernova shell center at each frequency. In the worse cases (i.e., smallest shell sizes), the standard deviation in the SN 1993J coordinates is around $10-20 \%$ of the supernova radius. Such a large shift in the supernova shell center could be easily appreciated by visual inspection in the images. We did not see such large deviations in the position of the supernova shell center at any of the epochs reported here. Additionally, even if such large shifts had taken place in the estimate of the supernova shell center, as determined from phase-referencing to $\mathrm{M} 81^{*}$, we conclude from simulations that the effect of such (random-like) shifts on the shell size would be on the order of $5 \%$, which translates into an effect of only $1 \%$, or lower, on the fitted parameters of the expansion curve reported in the next section.

\subsection{Expansion curve}

In this section, we report on the combined analysis of the available VLBI data with different approaches. On the one hand, we applied a novel analysis method to the data in the sky plane (the common-point method, CPM, described in Martí-Vidal 2008; and Appendix A of Marcaide et al. 2009). This method relies on some mathematical properties of the convolution of a Gaussian

\footnotetext{
${ }^{5}$ For the observations that were not phase-referenced to M 81*, we used the shell center estimated from model fitting.
} 
with an image of a source with azimuthal symmetry. It can be shown that there are points in the azimuthal average of the convolved image that do not change under first-order modifications of the width of the convolving Gaussian. These points can be related to the size of the source.

On the other hand, we used model fitting to the visibilities, following the same approach as in Marcaide et al. (2009) (see their Sect. 4.4, and their Appendix B). This approach for model fitting has some advantages, compared to the approach followed by Bartel et al. (2002) (see Sect. 8 of Marcaide et al. 2009, for more details). Then, we check the similarities and discrepancies of the expansion curves obtained with both approaches.

\subsubsection{Expansion with the common-point method}

The CPM analysis of all the images of SN 1993J obtained as described in Sect. 2, yields the shell radii shown in Table 1, Col. 5 (we call these size estimates $R_{\mathrm{SC}}$ ). For epochs earlier than 20 September 1994, the supernova sizes cannot be measured well with the CPM. It would imply over-resolution of the images. Indeed, the convolving beam after applying the CPM to the data of epoch 23 February 1995 is 0.74 mas, which is similar to, but still slightly smaller than, the corresponding interferometric beam ( $~ 0.94$ mas using uniform weighting). Therefore, for data observed before 1995, we used the shell sizes estimated from model fitting to the visibilities. For epochs of Bartel's group earlier than year 1995, we used their results published in Bartel et al. 2002. These sizes were then transformed into "CPM sizes" by applying the corresponding factors, which were obtained theoretically from numerical simulations. For a shell with a fractional width of 0.3 and maximum absorption from the ejecta (i.e., the emission structure that we assume for SN 1993J), the ratio of model-fitting to $\mathrm{CPM}$ size $^{6}$ is 1.031 , provided the model used to fit the visibilities does not take ejecta absorption into account and has a fractional width of only 0.2 (i.e., the model used in Bartel et al. 2002). In any case, all these transformation factors are always close to unity. Indeed, their deviations from 1 are similar to the relative uncertainties of the shell-size estimates.

Also, for very early epochs, when the synchrotron selfabsorption is large, a shell profile is not able to properly model the supernova structure, since the emission pattern of a spherically symmetric optically thick source is disk-like. Moreover, the transition from optically thick to optically thin for the synchrotron radiation, is frequency-dependent. Therefore, we expect to have some frequency-dependent biases in the expansion curve determined for very early epochs (earlier than day 100-200 after explosion). However, these few data do not affect our results above the $0.5 \sigma$ level, given the long time coverage and dense sampling of the expansion curve.

Figure 3(a) (plot on a linear scale) and (b) (plot on a logarithmic scale) shows several weighted-least-square fits of the supernova radius, $R$, to power laws of time. The last four epochs were excluded from the fits, since they clearly depart from the general behavior. The fit shown as a continuous line uses all data at $22,15,8.4$, and $5 \mathrm{GHz}$, and fits a model with 4 parameters: two expansion indices $\left(m_{1}\right.$ and $\left.m_{2}\right)$, a break time ( $t_{\mathrm{br}}$, that separates the expansion regimes given by each expansion index), and

\footnotetext{
${ }_{6}$ This factor is computed for a shell with radius of 0.6 mas, observed at the frequency of $5 \mathrm{GHz}$. This factor slightly depends on the shell size and/or on the $u v$-coverage.
}

the supernova size at the break time $\left(r_{\mathrm{br}}\right)$. The expression for the fitted model is

$R(t)=\left\{\begin{array}{c}r_{\mathrm{br}}\left(t / t_{\mathrm{br}}\right)^{m_{1}}, \quad t<t_{\mathrm{br}} \\ r_{\mathrm{br}}\left(t / t_{\mathrm{br}}\right)^{m_{2}}, \quad t \geq t_{\mathrm{br}}\end{array}\right.$

This model is the same as the one used by Marcaide et al. (2009) to fit their data at 8.4 and $5 \mathrm{GHz}$. The uncertainties of the measured radii were uniformly scaled to make the reduced $\chi^{2}$ equal to unity (the resulting uncertainties are those shown in Table 1 Col. 5). The results of the fit to data at $22,15,8.4$, and $5 \mathrm{GHz}$ are shown in Table 2, row 1. Adding the data at $2.3 \mathrm{GHz}$ to the fit does not change the results above the $1 \sigma$ level.

Since we adapted the published (model fitting) size estimates to CPM size estimates for epochs earlier than day 541, one could suspect that the fitted break time (and, consequently, also $m_{1}$ and $m_{2}$ ) could be strongly affected by the conversion factors applied to the model-fitting sizes, in order to convert them into CPM sizes. This is not the case; indeed, since using very different emission models (shell widths ranging from 0.2 to 0.3 and/or different levels of ejecta opacity, from $100 \%$ to $0 \%$ ) translates into deviations in the modelfit-to-CPM conversion factors of $\sim 3 \%$, we multiplied all the sizes of epochs earlier than day 541 first by 0.97 , and later by 1.03 , and each time re-fitted the resulting expansion curves. The resulting expansion parameters are compatible each time to those shown in Table 2, row 1, at a $\sim 0.4 \sigma$ level (for $t_{\mathrm{br}}$ ), $\sim 0.2 \sigma$ level (for $m_{1}$ ), and at a $\sim 1.2 \sigma$ level (for $m_{2}$ ). The expansion parameters shown in Table 2 are, therefore, very insensitive to the conversion factors applied to the (early) modelfitting sizes.

The fit shown in Fig. 3 (dashed line) uses data only at $1.7 \mathrm{GHz}$, and the simple model given by equation

$R(t) \propto t^{m_{3}}$.

The uncertainties of the measured radii were also scaled to make the reduced $\chi^{2}$ equal to unity. The result of the fit to only the 1.7 GHz data is shown in Table 2, row 1 .

To quantify the evidence of the frequency-dependent expansion described in Marcaide et al. (2009) and in this paper, we used the model given by Eq. (2) to fit the size estimates at $5 \mathrm{GHz}$ for epochs later than day 2000 (i.e., roughly the supernova age at the first $1.7 \mathrm{GHz}$ epoch). The resulting expansion index at $5 \mathrm{GHz}$ is $m=0.771 \pm 0.016$, which is at $\sim 2.8 \sigma$ from the expansion index determined at $1.7 \mathrm{GHz}$ in the same time range $(m=0.87 \pm 0.02)$.

We also applied the CPM to the supernova images obtained just after the phase-reference calibration (i.e., without any phase self-calibration). The resulting expansion curve and fitted parameters are shown in Fig. 4(a) and Table 2 (row 2), respectively. We notice that these results are compatible with those given just above.

We should also point at that even though these fits describe the supernova expansion satisfactorily for most of the observations, for epochs observed after day 3500-4000 the sizes at all frequencies are systematically smaller than the model predictions, as can be seen in Figs. 3 and 4. Even then, the observed size at $1.7 \mathrm{GHz}$ on day 4606 after explosion is larger than the size at $5 \mathrm{GHz}$ observed on day 4591. In Paper II, we will discuss this "enhanced" deceleration of the supernova shell at very late epochs and its relationship with the exponential drop of the radio light curves reported by Weiler et al. (2007). 
Table 2. Expansion parameters of SN 1993J.

\begin{tabular}{l|ccc|c}
\hline \hline & \multicolumn{3}{|c|}{5 to $22 \mathrm{GHz}$} & $1.7 \mathrm{GHz}$ \\
& $m_{1}$ & $m_{2}$ & $t_{\text {br }}$ (days) & $m_{3}$ \\
\hline CPM selfcal $^{a}$ & $0.933 \pm 0.010$ & $0.796 \pm 0.005$ & $390 \pm 30$ & $0.87 \pm 0.02$ \\
CPM ph-ref $^{b}$ & $0.933 \pm 0.010$ & $0.795 \pm 0.005$ & $390 \pm 40$ & $0.83 \pm 0.02$ \\
Model fitting $^{c}$ & $0.94 \pm 0.06$ & $0.798 \pm 0.007$ & $270 \pm 70$ & $0.90 \pm 0.03$ \\
Bartel 1 $^{d}$ & $0.93 \pm 0.02$ & $0.798 \pm 0.006$ & $390 \pm 50$ & $0.84 \pm 0.06$ \\
Bartel $^{e}$ & $0.82 \pm 0.03$ & $0.796 \pm 0.016$ & $1000 \pm 700$ & $0.84 \pm 0.06$ \\
Marcaide $^{f}$ & $0.845 \pm 0.005$ & $0.788 \pm 0.015$ & $1500 \pm 300$ & $0.87 \pm 0.03$ \\
\hline
\end{tabular}

Notes. ${ }^{(a)}$ Using CPM-measured supernova sizes from images obtained from self-calibrated visibilities, as described in Marcaide et al. (2009). ${ }^{(b)}$ Using CPM-measured sizes from images obtained from phase-referenced visibilities. ${ }^{(c)}$ Using model fitting to the visibilities. ${ }^{(d)}$ Using the shell sizes reported in Bartel et al. (2002). ${ }^{(e)}$ Using the sizes reported in Bartel et al. (2002), but taking only the epochs later than day 182 after explosion (i.e., the day of the first epoch reported in Marcaide et al. 2009). ${ }^{(f)}$ Refit using the shell sizes reported in Marcaide et al. (2009).

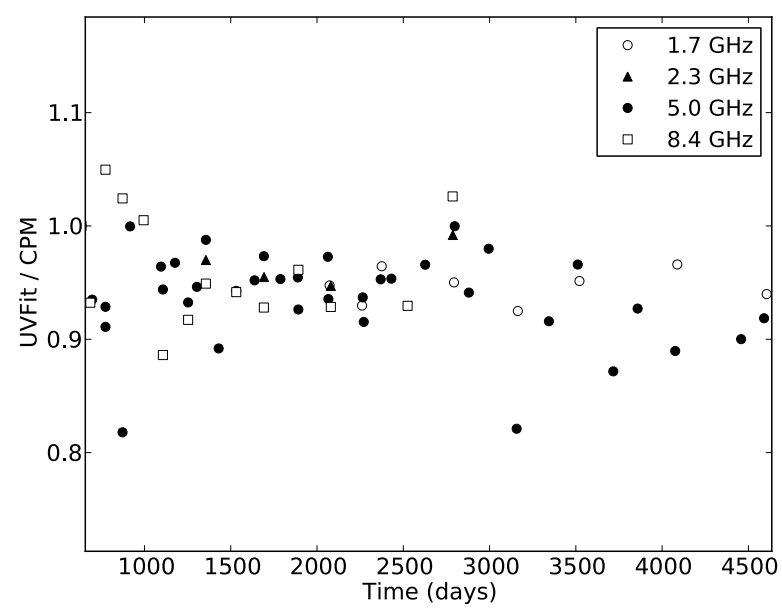

Fig. 2. $R_{\mathrm{MF}} / R_{\mathrm{SC}}$, i.e., ratios of sizes estimated from visibility model fitting $\left(R_{\mathrm{MF}}\right.$, see Sect. 3.2.2) to sizes obtained with the CPM $\left(R_{\mathrm{SC}}\right.$, see Sect. 3.2.1).

\subsubsection{Expansion from analysis in Fourier space}

If we fit a partially-absorbed shell model (the model used in Sect. 3.1 and described in Marcaide et al. 2009, Appendix B) to the $22,15,8.4$, and $5 \mathrm{GHz}$ visibilities obtained after the calibration described in Sect. 2 (we call these size estimates $R_{\mathrm{MF}}$ ), the resulting expansion curve is the one shown in Fig. 4 (data of day 4235 at $5 \mathrm{GHz}$ could not be properly modeled). The fitted parameters are shown in Row 3 of Table 2. Adding the data at $2.3 \mathrm{GHz}$ to the fit does not change these results above the $1 \sigma$ level. The result of the fit to only the $1.7 \mathrm{GHz}$ data is also shown in Row 3 of Table 2.

The parameters fitted to the expansion curve determined with the CPM are compatible with those of the expansion curve obtained from visibility model fitting, although the scatter in the latter expansion curve is higher. In Fig. 2, we show the ratios of sizes estimated from visibility model fitting to those estimated with the CPM. These ratios are $\sim 0.95$, and the scatter in this plot is mainly due to the scatter in the sizes estimated from visibility model fitting. The ratios at $1.7 \mathrm{GHz}$ are less scattered, and the ratios at $5 \mathrm{GHz}$ seem to be slightly lower at later epochs. This is an expected result if the different sizes at different frequencies are due to different radial intensity profiles of the supernova (see Sect. 3.4.2). Since the effect of different intensity profiles is
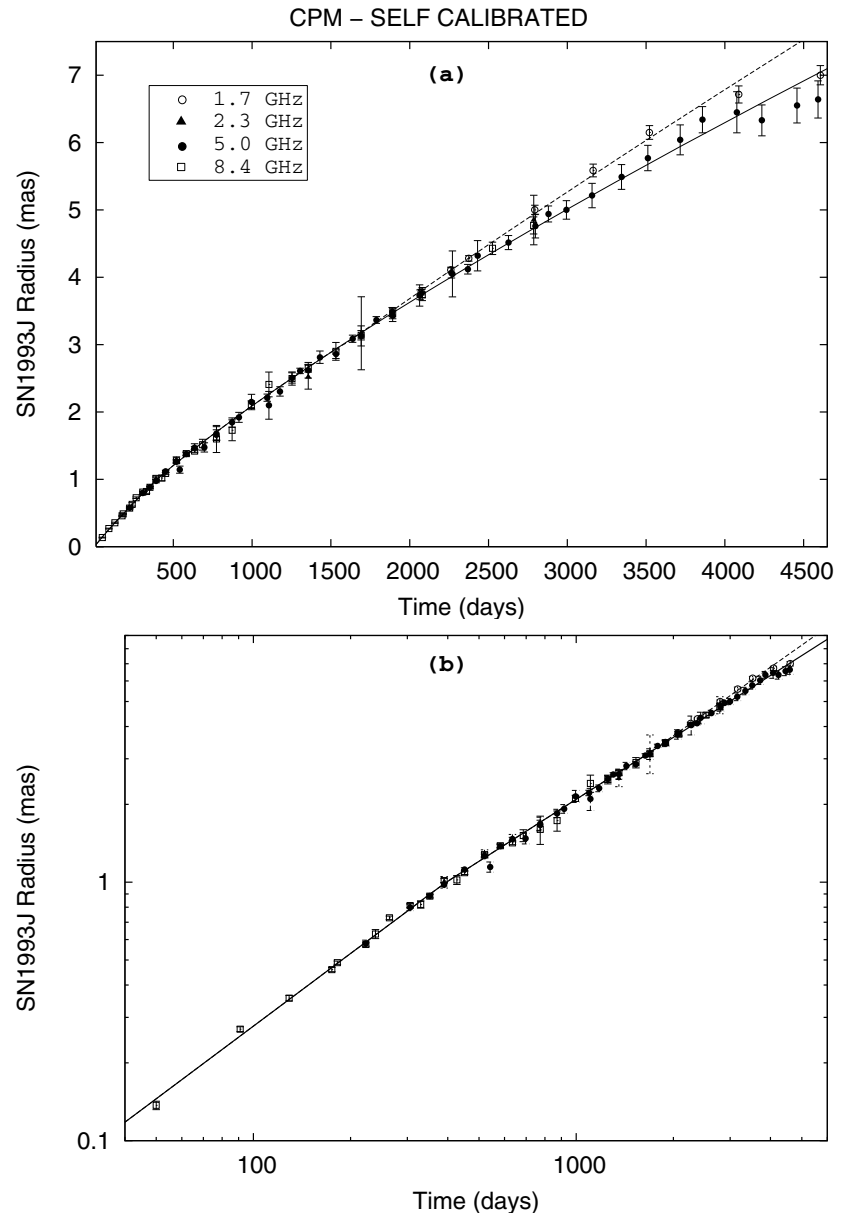

Fig. 3. a) Expansion of supernova SN 1993J. The radii were measured with the CPM applied to the images obtained from the process described in Sect. 2 (we call these sizes $R_{\mathrm{SC}}$ ). The dashed line corresponds to the model given by Eq. (2), fitted to only the $1.7 \mathrm{GHz}$ data. The continuous line is the model given by Eq. (1), fitted to the data at higher frequencies. b) Same as in a), but in logarithmic scale.

lower for the CPM sizes than for the model-fitting sizes (i.e., the CPM size estimates are more insensitive to changes in the radial intensity profile of the source, see Appendix A of Marcaide et al. 2009), any systematic effect in the data towards a smaller size estimate (like a decrease in the ejecta opacity) should decrease the ratio of model-fitting sizes to CPM sizes. 

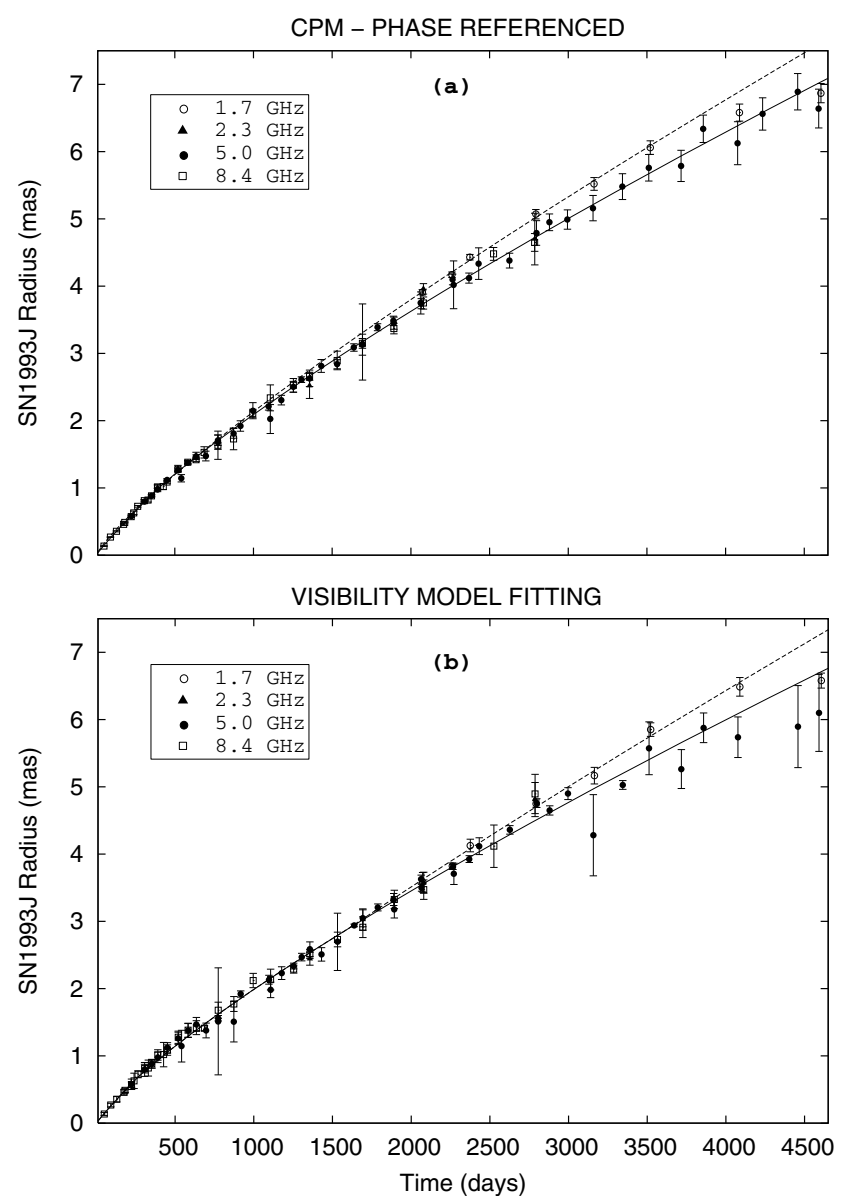

Fig. 4. a) Same as in Fig. 3a, but with radii measured with the CPM applied to the images obtained from the phase-referenced visibilities (we call these size estimates $R_{\mathrm{PR}}$ ). b) Same as in Fig. 3a, but with radii estimated from model fitting to the visibilities (we call these estimates $R_{\mathrm{MF}}$ ).

\subsection{Comparison of the different expansion models}

In Table 2 we summarize the results of fitting the models given by Eqs. (1) and (2) to the expansion curves of SN 1993J, obtained with different approaches and different time coverages. The results shown in the first three rows of Table 2 correspond to fits using the complete set of VLBI data here reported, analyzed following the approaches described in the previous sections. The first row corresponds to the sizes determined with the CPM, applied to the supernova images obtained following the special self-calibration described in Marcaide et al. (2009). The analysis procedure described in that publication has passed several tests with synthetic data and has been shown to give more precise size estimates than model fitting to the visibilities. Therefore, the expansion model that we consider definitive for SN 1993J is the one corresponding to the first row of Table 2.

The fitted parameters in the fourth row correspond to the supernova sizes published in Bartel et al. (2002). These parameters are very close to the results reported here for the complete set of observations (rows 1, 2, and 3). Therefore, even though the conclusions of Bartel et al. (2002) are very different from ours, their fitted shell sizes are compatible with our expansion model, i.e., one expansion regime for the $1.7 \mathrm{GHz}$ data and two expansion regimes (separated by a break time) for the higher frequencies. Indeed, the sizes at $1.7 \mathrm{GHz}$ reported in Bartel et al. (2002) are up to $5 \%$ larger than those reported by the same authors at the higher frequencies. In our analysis, the maximum size difference between 1.7 and $5 \mathrm{GHz}$ is $\sim 7.3 \%$ (observations of November 2001).

The last two rows of Table 2 correspond to fits of the subset of observations reported in Marcaide et al. (2009) and the subset of observations reported by Bartel et al. (2002), but taking only those from day 182 after explosion onwards (for a direct comparison of the models fitted to the data reported in these two works). We note that Marcaide et al. (2009) claim that the sizes at $1.7 \mathrm{GHz}$ can be modeled by an extrapolation of the early expansion curve (i.e., that with the expansion index $m_{1}=0.845$ ). Thus, a fit to the $1.7 \mathrm{GHz}$ sizes, alone, was not reported in Marcaide et al. (2009). Fitting those $1.7 \mathrm{GHz}$ sizes using Eq. (2) results in $m_{3}=0.87$ (Table 2, row 6), which is within $1 \sigma$ from $m_{1}$.

As can be seen, the fits shown in the first four rows are very similar. The results in the last two rows differ from the other ones mainly in the estimates of the break time (which also has large statistical uncertainties) and of $m_{1}$ (which is $\sim 9 \%$ lower). Clearly, the poor early time coverage of the subsets of observations corresponding to the fits of rows 5 and 6 of Table 2 results in a lower $m_{1}$, which in turn results in a later $t_{\mathrm{br}}$ to adequately model the later supernova expansion.

We could try to fit our complete expansion curve to a model with two break times, to check whether the break of day 1500 reported by Marcaide et al. (2009) (and reproduced here in row 6 of Table 2) can or cannot be recovered from the analysis of the whole data set. However, we find from Monte Carlo simulations that fitting such a model with two breaks to the data would not give reliable results (as long as such breaks are left as free parameters in the fit). Therefore, it is difficult to conclude whether both breaks are present in the data or not. We notice, however, that Marcaide et al. (2009) interpret the break time reported at day 1500 , not as a real break in the expansion curve, but as the result of several effects (related to the ejecta opacity and/or to a possible radial drop in the amplified magnetic fields inside the emitting region), which are strong enough to affect the measured expansion curve at the higher frequencies, but not at $1.7 \mathrm{GHz}$. This interpretation of the expansion curve, taking also the early break at day 400 into consideration, will be analyzed in Paper II.

\subsection{Structure evolution of SN 1993J}

\subsubsection{Estimates of ejecta opacity and shell width}

We measured the supernova shell radius using a method unrelated to model fitting in Fourier space (i.e., the CPM). Thus, we can use these measured sizes as fixed parameters in a model fitting, in which we can estimate the fractional shell width. Given that the CPM has a small bias, dependent on the emission structure of the supernova (see Marcaide et al. 2009), we should correct the shell sizes with the right bias before estimating the shell width. However, the bias of the CPM depends on the degree of absorption by the ejecta, and also on the fractional shell width, which is the quantity to be determined from model fitting. In short, we have a coupling between fitted shell widths and CPM biases. We can look for self-consistency in that coupling, finding a shell width for which the bias of the CPM, applied to the (fixed) shell sizes in the model fitting, translates into a fitted shell width corresponding to the CPM bias already applied. See Sects. 6.2 and 7.2 of Marcaide et al. 2009 for a detailed description of the trial-error procedure to find this self-consistency. Such a selfconsistent fractional shell width is $\sim 0.35$, for a model with maximum (i.e., $100 \%$ ) ejecta opacity. 


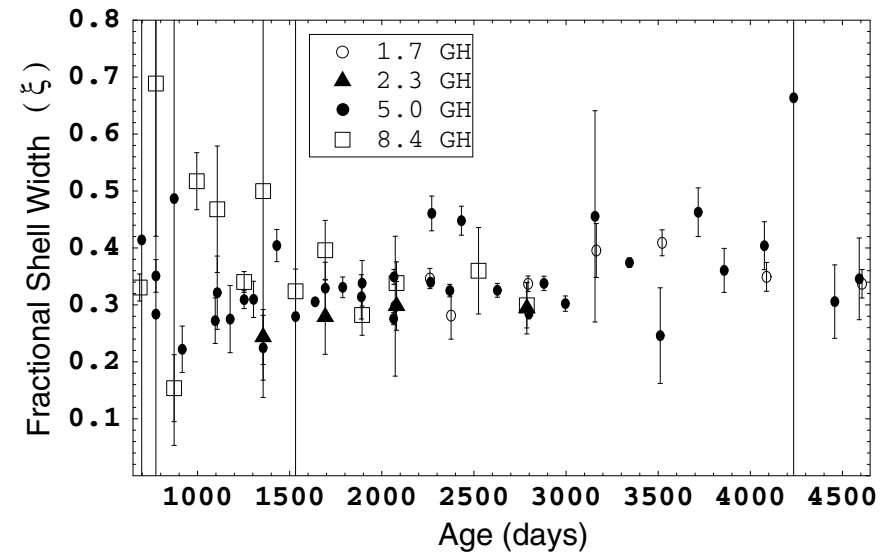

Fig. 5. Fitted fractional shell widths of SN 1993J, using the model described in Appendix 2 of Marcaide et al. (2009) and the shell sizes determined with the CPM, applying the corresponding bias for an ejecta absorption of $80 \%$ (see text). The errors shown are such that the reduced $\chi^{2}$ of each fit is equal to unity.

The percentage of ejecta opacity could be somewhat lower than $100 \%$ and/or could evolve in time. When we require selfconsistency between (bias-corrected) CPM results and (modelfitted) shell widths, lower ejecta opacities translate into narrower shell widths. In that sense, a value of 0.35 can be considered as an observational upper bound of the fractional shell width of SN 1993J.

An appropriate percentage of absorption to use in our fits is the average of the estimates obtained from fitting a shell model to the data of our best epochs (i.e., with good $u v$-coverages and large signal-to-noise ratios). The epochs selected for such fits were all at $5 \mathrm{GHz}$ between days 1638 and 2369 after explosion. We used data only at $5 \mathrm{GHz}$ to avoid any possible frequency-dependent bias. For all 10 epochs, we fitted the fractional shell width, $\xi=\left(R_{\text {out }}-R_{\text {in }}\right) / R_{\text {out }}$, the supernova radius, $R_{\text {out }}$, the location of the shell center, the percentage of absorption, and the total flux density, obtaining an average relative shell width of $0.31 \pm 0.02$ and an average percentage of absorption of $(80 \pm 8) \%$. These results are compatible with those reported in Marcaide et al. (2009), who used a subset of the observations presented here.

For an ejecta opacity of $80 \%$, the shell widths obtained from model fitting are shown in Fig. 5. Only observations of epochs from year 1995 onwards were used in the fitting. Using these values, we obtain a weighted mean of the shell width of $0.310 \pm 0.011$ for $8.4 \mathrm{GHz}$ data, $0.300 \pm 0.005$ for $5 \mathrm{GHz}$ data, $0.26 \pm 0.02$ for $2.3 \mathrm{GHz}$ data, and $0.324 \pm 0.008$ for $1.7 \mathrm{GHz}$ data.

All these quantities are close to 0.3. Based on very few and noisy data, the shell widths estimated at $2.3 \mathrm{GHz}$ are the smallest, within $2 \sigma$ from the value 0.3 . On the other hand, data at 8.4 and $1.7 \mathrm{GHz}$ give wider shell width estimates than at $5 \mathrm{GHz}$. The shell width at $8.4 \mathrm{GHz}$ is compatible with that at $5 \mathrm{GHz}$ at a $1 \sigma$ level. The average shell width at $1.7 \mathrm{GHz}$ is about $2 \sigma$ wider than at $5 \mathrm{GHz}$. The difference between shell widths at these frequencies could be due to either a physically wider shell at $1.7 \mathrm{GHz}$, to instrumental effects related to the finite sensitivity of the interferometers, or to a lower ejecta opacity at $5 \mathrm{GHz}$ (lower opacities translate into narrower fitted shell widths). Any of these explanations (or a combination of them) could help explain the different shell widths obtained for different frequencies (see Marcaide et al. 2009). In Paper II, we will analyze these possibilities and

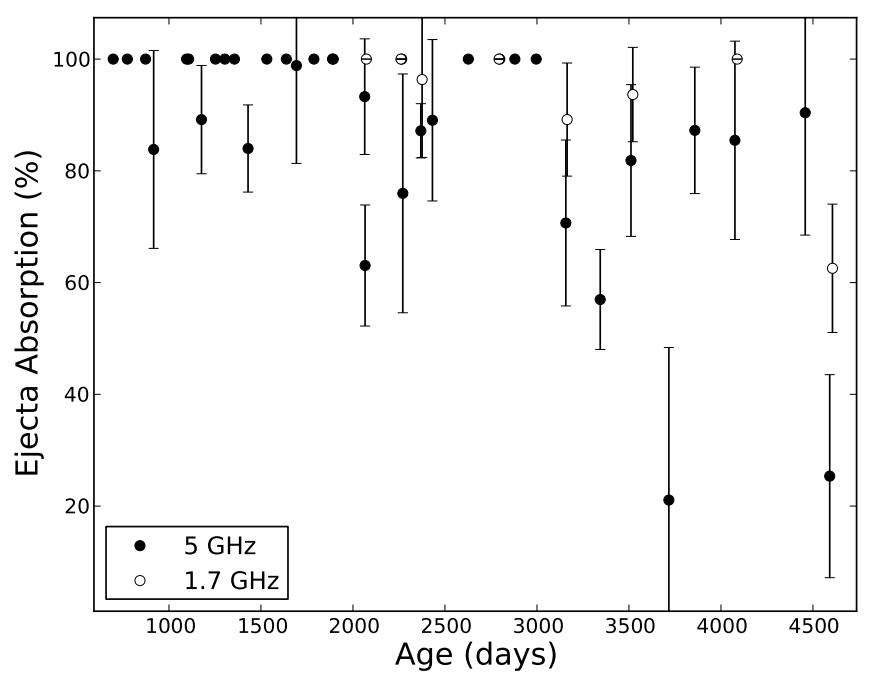

Fig. 6. Percentage of absorption (or degree of ejecta opacity) fitted to the visibilities by fixing the shell size and the fractional shell width (see text). The maximum allowed absorption (or ejecta opacity) in the model is set to $100 \%$.

their relationship with features in the radio light curves published by Weiler et al. (2007). From Fig. 5, we also notice that no time evolution of the relative shell width is discernible at any frequency, although the data are too noisy to reach any robust conclusion.

For completeness, we fitted the percentage of absorption at $5 \mathrm{GHz}$ and $1.7 \mathrm{GHz}$ by fixing the fractional shell width to 0.3 and the shell sizes to the estimates given by Eq. (2) (according to our hypothesis of different ejecta absorptions at different frequencies, this equation should give the closest estimates of the true shell size at late epochs). The results obtained are shown in Fig. 6. We set the maximum possible absorption to $100 \%$, to obtain fits with physical meaning for some epochs. There is a hint of a larger absorption at $1.7 \mathrm{GHz}$ compared to that at $5.0 \mathrm{GHz}$, although the data are too noisy to infer any clear evolution in the opacity.

\subsubsection{Spectral-index gradients in the shell}

Since the expansion curve of SN 1993J is dependent on the observing frequency, there must be a distribution of spectral indices through the shell. If the size at $1.7 \mathrm{GHz}$ is really larger than at $5 \mathrm{GHz}$, the spectral index in the outer part of the shell should tend to $-\infty$, since there would be emission at $1.7 \mathrm{GHz}$ but not at $5 \mathrm{GHz}$. On the contrary, if the frequency effects in the expansion curve came from changes in the opacity by the ejecta, the spectral indices in the inner part of the shell would be larger than those in the outer shell (since the intensity at $5 \mathrm{GHz}$ in the inner shell would be higher, because of the lower ejecta opacity).

Unfortunately, the spectral-index images of SN 1993J between 1.7 and $5 \mathrm{GHz}$ are very noisy. No clear conclusion can be extracted from the images themselves. However, we can increase the SNR of the spectral-index estimates by integrating the intensity at each frequency through different parts of the shell. On the other hand, we integrate the flux densities from a radial distance of 0 (i.e., the shell center) up to half the shell size at $1.7 \mathrm{GHz}$; we call these integrated flux densities $F_{50}^{\text {in }}$ and $F_{17}^{\text {in }}$, for the images at 5.0 and $1.7 \mathrm{GHz}$, respectively. On the other hand, 


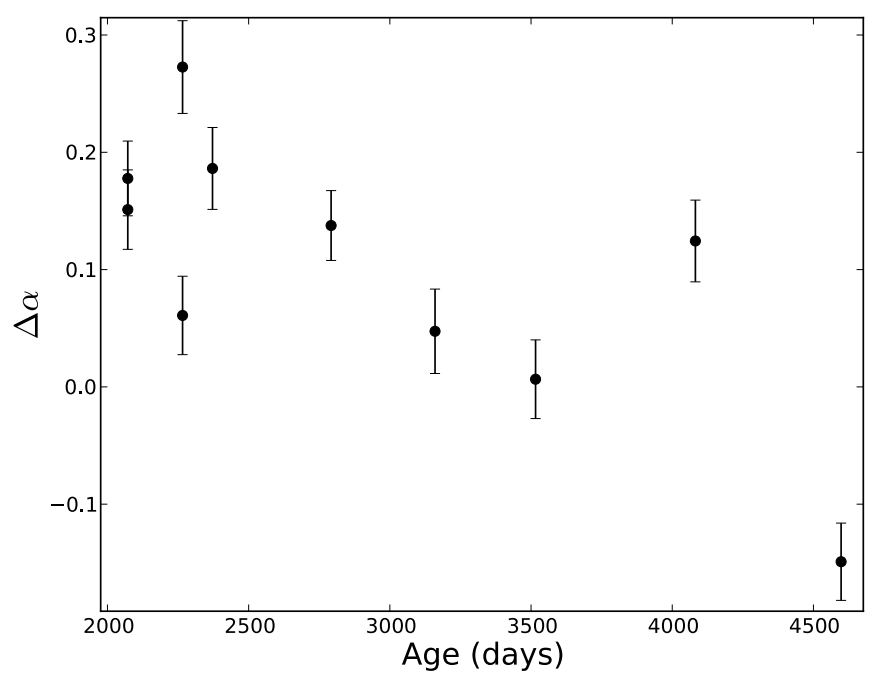

Fig. 7. Difference between the $1.7-5 \mathrm{GHz}$ spectral index in the inner shell (i.e., for radii up to 0.5 times the shell radius) and in the outer shell (i.e., for radii larger than 0.5 times the shell radius).

we integrate the flux densities from a radial distance of 0.5 times the shell size at $1.7 \mathrm{GHz}$ up to 2 times the shell size at $1.7 \mathrm{GHz}$ (to be sure that we integrate all the emission from the outer shell at both frequencies); we call these integrated flux densities $F_{5.0}^{\text {out }}$ and $F_{1.7}^{\text {out }}$, for $5 \mathrm{GHz}$ and $1.7 \mathrm{GHz}$, respectively. In Fig. 7 we show the difference between the inner spectral index,

$\alpha_{\text {in }}=\log \left(\frac{F_{5.0}^{\text {in }}}{F_{1.7}^{\text {in }}}\right) / \log \left(\frac{5.0}{1.7}\right)$,

and the outer spectral index,

$\alpha_{\text {out }}=\log \left(\frac{F_{5.0}^{\text {out }}}{F_{1.7}^{\text {out }}}\right) / \log \left(\frac{5.0}{1.7}\right)$.

We call this difference $\Delta \alpha=\alpha_{\text {in }}-\alpha_{\text {out }}$. It can be seen in the figure that $\Delta \alpha$ is positive for all the epochs where quasi-simultaneous 1.7 and $5 \mathrm{GHz}$ observations were available (except for the last one, at day 4606 after explosion). This is independent observational evidence of the opacity effects by the ejecta suggested in Marcaide et al. (2009) and supported here. The values of $\Delta \alpha$ at the earlier epochs gather around $0.1-0.3$. The expected value of $\Delta \alpha$ for a shell model with a fractional width of 0.3 and zero absorption at $5 \mathrm{GHz}$ (and maximum at $1.7 \mathrm{GHz}$ ) is 0.4 , a value higher than those shown in Fig. 7. It should be noticed that the presence of hot spots in the outer shell and/or changes in the ejecta opacity through the inner shell at each frequency (and, of course, the different sampling of Fourier space between both frequencies) may affect the estimates of $\Delta \alpha$.

\subsubsection{Azimuthal evolution of the shell inhomogeneities}

The VLBI images of SN 1993J keep a high degree of circularity during the whole expansion. A quantitative representation of the degree of circularity of the shell is the fractional uncertainty of the radius determined with the CPM (i.e., the scatter of radial positions of a given contour with respect to the shell center, in units of the source radius; see Appendix 1 of Marcaide et al. 2009 for more details). The degree of circularity of the supernova, computed this way, is typically around $2-4 \%$, as can be

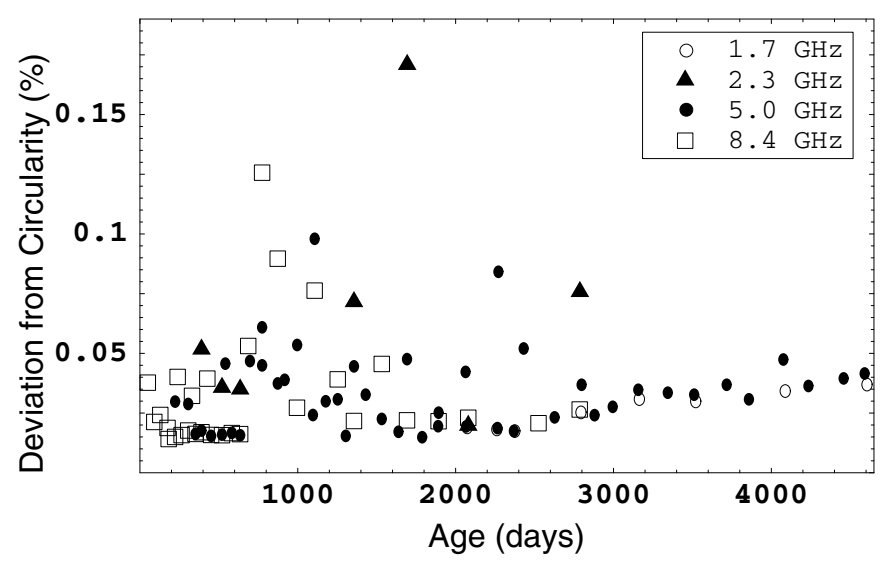

Fig. 8. Degree of circularity of the SN 1993J shell, as indicated by the fractional uncertainty in the source radius estimates when determined with the CPM (see Appendix 1 of Marcaide et al. 2009).

seen in Fig. 8, with the exception of some epochs with low dynamic ranges. Similar results were also reported in Bietenholz et al. (2001). Such a circularity in the images must be due to a high degree of isotropy in the angular distribution of the ejecta velocities (and, therefore, in the distribution of the CSM).

However, the symmetry of the radio shell not only depends on its circularity, but also on the intensity distribution inside it. To study the azimuthal intensity distribution in the shell, we computed, for each epoch since 1995, the angular distribution of flux density in a ring of radius equal to the radial position of the brightness peak. For every epoch, we used a convolving beam with $F W H M$ equal to 0.5 times the shell radius. In Fig. 9 we show the time evolution of the angular intensity distribution in the SN 1993J shell, obtained from a linear interpolation between epochs. In the cases of epochs observed less than 50 days apart, we selected only one for the interpolation, that of highest dynamic range ${ }^{7}$. The minimum-to-maximum intensity ratio at each epoch typically ranges between 0.7 and 0.9 , indicating that the shell emission is homogeneous to a level of $\sim 80 \%$.

From Fig. 9 we arrive at a clear conclusion: there are some regions where the shell is clearly brighter (i.e., has hot spots), and these regions persist in time for periods of the order of a thousand days. Unfortunately, the dynamic range of the images is not high enough to ensure a single interpretation of the azimuthal evolution of the radio shell. The first hot spot is located in the west (i.e., position angle of 270 degrees in Fig. 9) and is present from the beginning of the interpolation up to day $\sim 1600$ after explosion. There is another, less clear, hot spot present during approximately the same time range, but located in the east (i.e., position angle of 90 degrees). This hot spot seems to decompose in several parts at some epochs, which drift towards north and/or south. Beginning on day 1600 after explosion, this second hot spot seems to be finally decomposed into two hot spots, one towards the south (reaching a position angle $\sim 160$ degrees) and the other towards the north (reaching a position angle $\sim 0$ degrees). These two new hot spots persist in time beyond day 3500 after explosion. From that epoch onwards, the dynamic range of our images is too low to reach to any robust conclusion about the evolution of the angular brightness pattern. It is remarkable that the first hot spot located at a position angle of 270 degrees disappears more or less at the same time

7 The images of all these epochs can be downloaded from http : //www . uv . es/radioast/aanda2010/sn93j-images . html. 
I. Martí-Vidal et al.: Radio emission of SN1993J: the complete picture. I.

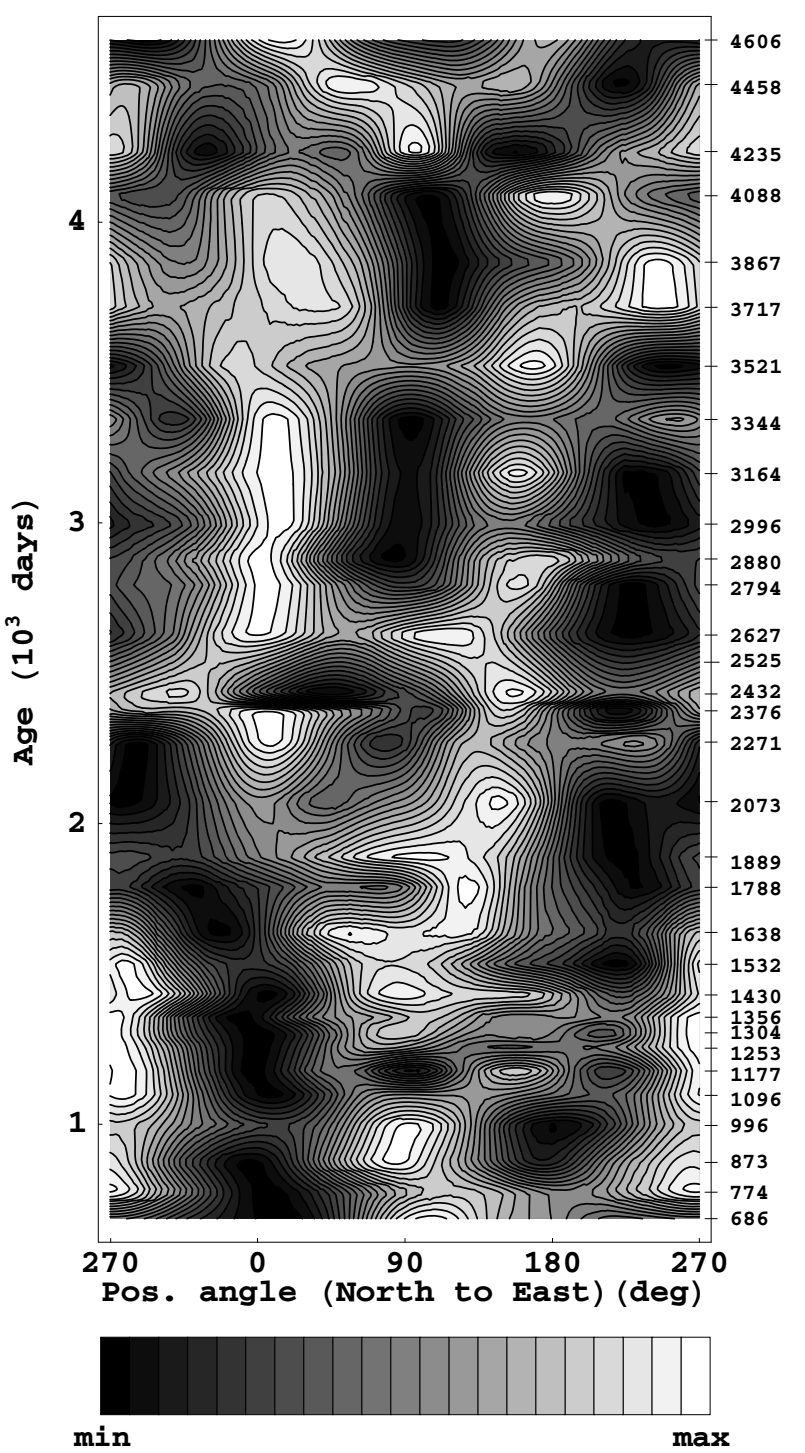

Fig. 9. Evolution of the azimuthal intensity distribution in the SN 1993J shell, computed as the linear time interpolation of a selection of epochs (see text). The supernova age at the selected epochs is marked with ticks (right). The 20 contours shown are normalized at each epoch, and equally distributed between the minimum (black) and maximum (white) intensities in the shell.

as the other hot spot evolves into two hot spots which shift to their final positions at 160 and 0 degrees. Another possible interpretation of Fig. 9 is that the hot spot located at 270 degrees could shift to 160 degrees on day $\sim 1600$, and the one located at 90 degrees could shift to $\sim 0$ degrees at roughly the same time. These interpretations of Fig. 9 differ from the evolution reported in Bietenholz et al. (2003). According to these authors, an additional hot spot should be visible at 180 degrees from day 774 to day 1258, which is not seen in Fig. 9. However, as we noticed above, for some epochs like that of day 1177, the wide hot spot at 90 degrees seems to decompose in several parts, and one of the parts shifts close to the south, thus making the figure compatible with the reported hot spot in Bietenholz et al. (2003). According to Bietenholz et al. (2003), another hot spot should also develop at 270 degrees, beginning on day 2080. A close look to Fig. 9 reveals that there are indeed small levels of over-emission between $\sim 200$ and $\sim 360$ degrees during practically the whole expansion. The additional hot spot reported in Bietenholz et al. (2003) could be related to some of the hot spots shown in our Fig. 9. We must notice, however, that Fig. 9 shows the intensity distribution along a circular ring of radius equal to that of the distance of the peak flux density to the shell center. Therefore, if a hotspot were at a distance from the shell center different from that of the peak, the azimuthal distribution shown in Fig. 9 would underestimate the intensity of this hot spot (and could also slightly missplace it), since the ring used in the azimuthal sampling would not cover the peak of such a hot spot.

The azimuthal intensity distribution in the shell can either come from inhomogeneities in the distribution of the magneticfield energy density (being higher at the regions with higher flux density) or from inhomogeneities in the CSM density distribution (being higher at the regions of higher flux density). However, we must take into account that the incomplete sampling of the $u v$-plane by the interferometers can also partially affect the recovered azimuthal intensity distributions, as explained in Heywood et al. (2009). Nevertheless, the clear systematic evolution of the features and the fact that they persist in the images even if we remove several antennas from the data (the hot spots are clearly encoded in the phase closures, which are independent of the antenna gains), give us confidence in the reliability of the results shown in Fig. 9.

In the case of magnetic-field inhomogeneities inside the shell, a variation in the angular distribution of the magnetic-field energy density would be due to anisotropies in the ejecta distribution, given that the magnetic fields are presumably amplified by nonlinear effects produced in the magneto-hydrodynamic interaction between the ejecta and the shocked CSM (see Chevalier 1982a). In the case of CSM inhomogeneities, an anisotropic pre-supernova stellar wind could help explain the hot spots reported here.

It must be noticed that the separation between the two hot spots that persist until day 1600 is approximately 180 degrees, which is approximately the same final angular separation of the two hot spots that develop after that day. Moreover, the angles between the location of the first hot spot (at 270 degrees) and the other hot spots that develop after day $\sim 1600$ are around 90 degrees. These peculiar angular separations could give clues to an interpretation of the hot spots as caused by an anisotropic pre-supernova stellar wind along the rotation axis and/or the equatorial plane of the progenitor. However, we do not consider the quality of Fig. 9 good enough to propose any such specific model.

\section{Summary}

We have re-analyzed all the available VLBI data of supernova SN 1993J in a homogeneous and self-consistent way. We find that the location of the supernova shell center, taking the location of the phase calibrator source $(\mathrm{M} 81 *)$ as a reference position, reflects the shift in the peak of emission of the calibrator with frequency (see also Bietenholz et al. 2004). We find no evidence of proper motion by the shell center at any frequency.

We have obtained expansion curves using two approaches in the data analysis: a novel method to estimate the shell size on the sky plane (the CPM, see Martí-Vidal 2008; and Marcaide et al. 2009) and model fitting to the visibilities. The expansion curves obtained with these approaches can be modeled with the same expansion law, and the fitted parameters obtained are compatible. As previously found by Marcaide et al. (2009), the expansion curve differs for different observing frequencies. For data at $1.7 \mathrm{GHz}$, we can model the expansion with only one expansion index, $m_{3}=0.87 \pm 0.02$. For data at the other, higher, frequencies, 
two expansion indices are needed, $m_{1}=0.933 \pm 0.010$ and $m_{2}=0.796 \pm 0.005$, which describe the expansion in two different regimes separated by a break time $t_{\mathrm{br}}=390 \pm 30$ days after explosion. These estimates are slightly different from those given by Marcaide et al. (2009) and are heavily influenced by having used of early data obtained by Bartel's group.

We estimated the shell width at different frequencies and its possible evolution with time, as well as the opacity to the radio emission by the ejecta. The mean fractional shell width found using data at all frequencies is $0.31 \pm 0.02$, compatible with the results previously reported in Marcaide et al. (1997) and Marcaide et al. (2009), but wider than the results reported by Bartel et al. (2000) and Bietenholz et al. (2003). Our study is not conclusive about any spectral dependence of the shell width or any time evolution. With regard to the ejecta absorption, we find that our best-quality data can be fitted with a partially-absorbed shell with $(80 \pm 8) \%$ absorption. We find evidence of a radial gradient in the distribution of the spectral index, indicating that the ejecta opacity may be different (higher) at $1.7 \mathrm{GHz}$, compared to that at higher frequencies, as pointed out in Marcaide et al. (2009). A more detailed study of the effect of a frequency-dependent ejecta opacity in the expansion curve and in the radio light curves is reported in Paper II.

We studied the morphological evolution of the radio shell beyond self-similarity. The inhomogeneities (hot spots) found around the azimuthal structure of the shell images are typically on the order of $20 \%$ of the mean flux density per unit beam in the shell. The hot spots found in the shell persist for times of the order of 1000 days and the angular separations between them usually take singular values ( $~ 90$ and/or $\sim 180$ degrees). This could be interpreted as the result of an anisotropic pre-supernova stellar wind along the rotation axis and/or the equatorial plane of the precursor star. However, the quality of the data is not good enough to reach any robust conclusion.

Shell sizes at very late epochs (from day 3500-4000 onwards) are systematically and progressively smaller than predicted with the expansion model. This effect may be related to the exponential-like time decay of the supernova flux density at late epochs reported in Weiler et al. (2007).

Acknowledgements. We thank the anonymous referee for his/her corrections, detailed analysis and good suggestions for improving the paper. I.M.V. is a fellow of the Alexander von Humboldt Foundation in Germany. The National Radio
Astronomy Observatory is a facility of the National Science Foundation operated under cooperative agreement with Associated Universities, Inc. The European VLBI Network is a joint facility of European,Chinese, South African and other radio astronomy institutes funded by their national research councils. Partial support from Spanish grant AYA 2005-08561-C03, AYA 2006-14986-C02, and Prometeo 2009/104 is acknowledged. A.A. and M.A.P.T. also acknowledge support by the Consejería de Innovación, Ciencia y Empresa of Junta de Andalucía through grants FQM-1747 and TIC-126.

\section{References}

Baron, E., Hauschildt, P. H., Branch, D., et al. 1995, ApJ, 441, 170 Bartel, N., Bietenholz, M. F., Rupen, M. P., et al. 1994, Nature, 368, 610 Bartel, N., Bietenholz, M. F., Rupen, M. P., et al. 2000, Science, 287, 112 Bartel, N., Bietenholz, M. F., Rupen, M. P., et al. 2002, ApJ, 581, 404 Bartel, N., Bietenholz, M. F., Rupen, M. P., \& Dwarkadas, V. V. 2007, ApJ, 668, 924

Bietenholz, M. F., Bartel, N., \& Rupen, M. P., 2001, ApJ, 557, 770

Bietenholz, M. F., Bartel, N., \& Rupen, M. P. 2003, ApJ, 597, 374

Bietenholz, M. F., Bartel, N., \& Rupen, M. P. 2004, ApJ, 615, 173

Blandford, R. D., \& Königl, A. 1979, ApJ, 232, 34

Chevalier, R. A. 1982a, ApJ, 258, 790

Chevalier, R. A. 1982b, ApJ, 259, 302

Fransson, C., \& Björnsson, C.-I. 1998, ApJ, 509, 861

Fransson, C., Lundqvist, P., \& Chevalier, R. A. 1996, ApJ, 461, 993

Freedman, W. L., Hughes, S. M., Madore, B. F., et al. 1994, ApJ, 427, 628

Heywood, I., Blundell, K. M., Klöckner, H.-R., \& Beasley, A. J. 2009, MNRAS, 392, 855

Kovalev, Y. Y., Lobanov, A. P., Pushkarev, A. B., \& Zensus, J. A. 2008, A\&A, 483, 759

Marcaide, J. M., \& Shapiro, I. I. 1983, AJ, 88, 1133

Marcaide, J. M., \& Shapiro, I. I. 1984, ApJ, 276, 56

Marcaide, J. M., Alberdi, A., Elósegui, P., et al. 1994, ApJ, 424, 25

Marcaide, J. M., Alberdi, A., Ros, E., et al. 1995, Nature, 373, 44

Marcaide, J. M., Alberdi, A., Ros, E., et al. 1997, ApJ, 486, 31

Marcaide, J. M., Martí-Vidal, I., Ros, E., et al. 2005, in Cosmic Explosions, ed.

J. M. Marcaide, \& K. W. Weiler (Berlin:Springer), IAU Colloq., 192, 29

Marcaide, J. M., Martí-Vidal, I., Alberdi, A., et al. 2009, A\&A, 505, 927

Martí-Vidal, I. 2008, Ph.D. Thesis (University of Valencia, Spain)

Martí-Vidal, I., Marcaide, J. M., Alberdi, A., et al. 2011, A\&A, 526, A143 (Paper II)

Mioduszewski, A. J., Dwarkadas, V. V., \& Ball, L. 2001, ApJ, 562, 869

Pérez-Torres, M. A., Alberdi, A., \& Marcaide, J. M. 2001, A\&A, 374, 997

Pooley, G. G., \& Green, D. A. 1993, MNRAS, 264, 17

Ripero, J., \& García, F. 1993, IAU Circ., 5731

Shepherd, M. C., Pearson, T. J., \& Taylor, G. B. 1995, BAAS, 26, 987

Van Dyk, S. D., Weiler, K. W., Sramek, R. A., Rupen, M. P., \& Panagia, N. 1994, ApJ, 432, L115

Weiler, K. W., Williams, C. L., Panagia, N., et al. 2007, ApJ, 671, 1959 